\title{
Bosonic string theory in background fields by canonical methods *
}

\author{
B. Sazdović ${ }^{\dagger}$ \\ Institute of Physics, 11001 Belgrade, P.O.Box 57, Serbia
}

June 6, 2018

\begin{abstract}
We investigate classical dynamics of the bosonic string in the background metric, antisymmetric and dilaton fields. We use canonical methods to find Hamiltonian in terms of energy-momentum tensor components. The later are secondary constraints of the theory. Due to the presence of the dilaton field the Virasoro generators have nonlinear realization. We find that, in the curve space-time, opposite chirality currents do not commute. As a consequence of the two-dimensional general covariance, the energy-momentum tensor components satisfy two Virasoro algebras, even in the curve space-time. We emphasize that background antisymmetric and dilaton fields are the origin of space-time torsion and space-time nonmetricity, respectively.
\end{abstract}

PACS number(s): 11.25.-w

\section{Introduction}

The string propagation in the flat Minkowski space-time has been investigated for a long time and it is well understood [1. The dynamics of string moving in arbitrary background metric, which can be interpreted as the nonlinear $\sigma$-model, has also been studied [2, 3, 4]. The goal of these investigations was to analyze the consistency of string dynamics in the presence of background fields. The condition for two-dimensional conformal invariance on the quantum level, which technically means vanishing of the renormalization group $\beta$ function for nonlinear $\sigma$-model, produce the field equations for the background fields. The quantization of the theory has been performed expanding the quantum fields $x^{\mu}$ around

\footnotetext{
${ }^{*}$ Work supported in part by the Serbian Ministry of Science, Technology and Development under contract No. 1486.

${ }^{\dagger}$ e-mail address: sazdovic@phy.bg.ac.yu
} 
the expectation value $x_{0}^{\mu}$, and using background field expansion by standard technique of Riemann normal coordinates. This remarkable connection between the quantum feature of the world-sheet (conformal invariance), and the classical space-time property of the string theory (equations of motion), has been obtained perturbatively.

In this paper, we are going to investigate string propagation in the arbitrary gravitational, antisymmetric tensor, and dilaton background fields. We will make classical nonperturbative investigation of the full nonlinear theory.

In Sec.2, we perform the canonical analysis, treating world-sheet fields as variables in the theory, and space-time fields as a background depending on the coordinates $x^{\mu}$. Because of the presence of the dilaton field, the conformal component of the world-sheet metric survives, and corresponding new currents appear. The energy-momentum tensor obtains the nonlinear realization. It is not in the Sugawara form, but is a more general bilinear combination of the currents, plus linear term in $\sigma$ derivative of the currents.

In Sec.3, Poisson bracket algebra of the currents and energy-momentum tensors components has been investigated. Let us stress that, in spite of the fact that some opposite chirality currents do not commute the energy-momentum tensors components, which appear as secondary constraints, satisfy two Virasoro algebras as a consequence of the world-sheet reparametrization invariance.

We also derive the Hamiltonian equations of motion, in Sec.4, using the PB relations between the energy-momentum tensor and the currents. With the help of Lagrangian expressions for the currents we easy convert the above Hamiltonian equations to the Lagrangian ones. Using these world-sheet equations of motion, we introduce the space-time connection and covariant derivative in the presence of the background fields.

In the conclusion, we discus the contribution of the antisymmetric tensor and the dilaton field to the space-time torsion and nonmetricity, respectively.

The Appendix A is devoted to the world-sheet geometry.

\section{Canonical analysis of the theory}

Let us consider bosonic string propagating in the non-trivial background, described by the action [1]-4

$$
S=\kappa \int_{\Sigma} d^{2} \xi \sqrt{-g}\left\{\left[\frac{1}{2} g^{\alpha \beta} G_{\mu \nu}(x)+\frac{\varepsilon^{\alpha \beta}}{\sqrt{-g}} B_{\mu \nu}(x)\right] \partial_{\alpha} x^{\mu} \partial_{\beta} x^{\nu}+\Phi(x) R^{(2)}\right\} .
$$

Here $\xi^{\alpha}(\alpha=0,1)$ are coordinates of two dimensional world-sheet $\Sigma$ and $x^{\mu}(\xi)(\mu=$ $0,1, \ldots, D-1)$ are coordinates of the $D$ dimensional space-time $M$. The background space-time fields: metric $G_{\mu \nu}$, antisymmetric tensor field $B_{\mu \nu}=-B_{\nu \mu}$ and dilaton field $\Phi$, depend on the coordinates $x^{\mu}$. The intrinsic world-sheet metric we denote by $g_{\alpha \beta}$ 
and corresponding scalar curvature by $R^{(2)}$. Through the paper we will use the notation $\partial_{\alpha} \equiv \frac{\partial}{\partial \xi^{\alpha}}$ and $\partial_{\mu} \equiv \frac{\partial}{\partial x^{\mu}}$.

If we parameterize the world-sheet metric tensor $g_{\alpha \beta}$ with the light-cone variables $\left(h^{+}, h^{-}, F\right)$ (for details see (A.1) and papers [5, 6]) and introduce the useful combination of background fields

$$
\Pi_{ \pm \mu \nu}=B_{\mu \nu} \pm \frac{1}{2} G_{\mu \nu}
$$

we can write the action in the form

$$
S=\kappa \int_{\Sigma} d^{2} \xi \sqrt{-\hat{g}}\left[2 \Pi_{+\mu \nu} \hat{\partial}_{+} x^{\mu} \hat{\partial}_{-} x^{\nu}+\Phi\left(\hat{R}^{(2)}+2 \hat{\Delta} F\right)\right]
$$

or more explicitly

$$
\begin{aligned}
S & =\kappa \int_{\Sigma} d^{2} \xi \frac{1}{h^{-}-h^{+}}\left[G_{\mu \nu}\left(\dot{x}^{\mu}+h^{-} x^{\mu \prime}\right)\left(\dot{x}^{\nu}+h^{+} x^{\nu \prime}\right)-2\left(h^{-}-h^{+}\right) B_{\mu \nu} \dot{x}^{\mu} x^{\nu \prime}\right. \\
& \left.+2\left(\dot{\Phi}+h^{-} \Phi^{\prime}\right)\left(\dot{F}+h^{+} F^{\prime}+h^{+\prime}\right)+2\left(\dot{\Phi}+h^{+} \Phi^{\prime}\right)\left(\dot{F}+h^{-} F^{\prime}+h^{-\prime}\right)\right] .
\end{aligned}
$$

Here in agreement with (A.4) we introduce light-cone derivatives $\hat{\partial}_{ \pm}=\frac{\sqrt{2}}{h^{-}-h^{+}}\left(\partial_{0}+h^{\mp} \partial_{1}\right)$. We also use the following notation $\dot{X}=\partial_{\tau} X$ and $X^{\prime}=\partial_{\sigma} X$ for any variable $X$.

The momenta corresponding to the fields $x^{\mu}, h^{ \pm}$and $F$ are

$$
\begin{aligned}
\pi_{\mu}=\frac{\partial \mathcal{L}}{\partial \dot{x}^{\mu}}= & \frac{\kappa}{h^{-}-h^{+}}\left\{G_{\mu \nu}\left[2 \dot{x}^{\nu}+\left(h^{-}+h^{+}\right) x^{\nu \prime}\right]-2\left(h^{-}-h^{+}\right) B_{\mu \nu} x^{\nu \prime}\right. \\
& \left.+2\left[2 \dot{F}+\left(h^{-}+h^{+}\right) F^{\prime}+\left(h^{-}+h^{+}\right)^{\prime}\right] \partial_{\mu} \Phi\right\} \\
\pi_{ \pm}=\frac{\partial \mathcal{L}}{\partial \dot{h}^{ \pm}}= & 0, \\
\pi_{F}=\frac{\partial \mathcal{L}}{\partial \dot{F}}= & \frac{2 \kappa}{h^{-}-h^{+}}\left[2 \dot{\Phi}+\left(h^{-}+h^{+}\right) \Phi^{\prime}\right] .
\end{aligned}
$$

From the eq. (2.7) we can solve the time derivatives of the field $\Phi$ as

$$
\dot{\Phi}=\frac{1}{4 \kappa}\left(h^{-} i_{-}^{\Phi}-h^{+} i_{+}^{\Phi}\right)
$$

where we introduced the currents

$$
i_{ \pm}^{\Phi}=\pi_{F} \pm 2 \kappa \Phi^{\prime}
$$

We will denote the gradient of the dilaton field $\Phi$ by $a_{\mu}$

$$
a_{\mu}=\partial_{\mu} \Phi, \quad a^{\mu}=G^{\mu \nu} a_{\mu}, \quad a^{2}=G^{\mu \nu} a_{\mu} a_{\nu} .
$$

We will suppose that the gradient of the dilaton field $\Phi(x)$ is not light-cone vector, and the condition $a^{2} \neq 0$ is fulfilled in the whole space-time.

Multiplying eq. (2.5) with $a^{\mu}$ we can solve it in terms of $\dot{F}$

$$
\dot{F}=\frac{1}{4 \kappa}\left(h^{-} i_{-}^{F}-h^{+} i_{+}^{F}\right)-\frac{1}{2}\left(h^{-}+h^{+}\right)^{\prime}
$$


where we define new currents

$$
i_{ \pm}^{F}=\frac{a^{\mu}}{a^{2}} j_{ \pm \mu}-\frac{1}{2 a^{2}} i_{ \pm}^{\Phi} \pm 2 \kappa F^{\prime}
$$

and

$$
j_{ \pm \mu}=\pi_{\mu}+2 \kappa \Pi_{ \pm \mu \nu} x^{\nu \prime}
$$

We used the relation $\dot{\Phi}=a_{\mu} \dot{x}^{\mu}$, which allow us to trade $\dot{x}^{\mu}$ for $\dot{\Phi}$. Substituting the expression for $\dot{F}$ back into (2.5), we can solve the same equation in terms of $\dot{x}^{\mu}$

$$
\dot{x}^{\mu}=\frac{G^{\mu \nu}}{2 \kappa}\left(h^{-} J_{-\nu}-h^{+} J_{+\nu}\right) .
$$

The corresponding currents $J_{ \pm \mu}$ are defined as

$$
J_{ \pm \mu}=P^{T}{ }_{\mu}^{\nu} j_{ \pm \nu}+\frac{a_{\mu}}{2 a^{2}} i_{ \pm}^{\Phi}=j_{ \pm \mu}-\frac{a_{\mu}}{a^{2}} j
$$

and we introduce the variable

$$
j=a^{\mu} j_{ \pm \mu}-\frac{1}{2} i_{ \pm}^{\Phi}=a^{2}\left(i_{ \pm}^{F} \mp 2 \kappa F^{\prime}\right) .
$$

The projection operator

$$
P_{\mu \nu}^{T}=G_{\mu \nu}-\frac{a_{\mu} a_{\nu}}{a^{2}}
$$

will arise frequently in this article. Let us explain its geometrical interpretation. Note that $a_{\mu}$ is a normal vector to the $D-1$ dimensional submanifold $M_{D-1}$, defined by the condition $\Phi(x)=$ const. For $a^{2} \neq 0$, the corresponding unit vector is $n_{\mu}=\frac{a_{\mu}}{\sqrt{\varepsilon a^{2}}}$. Here $\varepsilon=1$ if $a_{\mu}$ is time like vector $\left(a^{2}>0\right)$, and $\varepsilon=-1$ if $a_{\mu}$ is space like vector $\left(a^{2}<0\right)$, so that $n^{2}=\varepsilon$. Consequently, we can interpret the expression

$$
P^{T}{ }_{\mu \nu}=G_{\mu \nu}-\varepsilon n_{\mu} n_{\nu} \equiv G_{\mu \nu}^{D-1}
$$

as a induced metric on $M_{D-1}$.

The time derivatives of the fields $\Phi, F$ and $x^{\mu}$ we expressed in terms of the corresponding currents (2.8), (2.11) and (2.14). The $\sigma$ derivative of the same fields we can express in terms of the same currents

$$
x^{\mu \prime}=\frac{G^{\mu \nu}}{2 \kappa}\left(J_{+\nu}-J_{-\nu}\right), \quad F^{\prime}=\frac{1}{4 \kappa}\left(i_{+}^{F}-i_{-}^{F}\right), \quad \Phi^{\prime}=\frac{1}{4 \kappa}\left(i_{+}^{\Phi}-i_{-}^{\Phi}\right) .
$$

Note that we introduce the momenta $\pi_{\mu}, \pi_{ \pm}$and $\pi_{F}$ for two dimensional fields $x^{\mu}, h^{ \pm}$ and $F$, respectively. We did not introduce momentum for dilaton background field $\Phi$, but for convenience we have the currents $i_{ \pm}^{\Phi}$ in term of which we expressed the $\dot{\Phi}$ and $\Phi^{\prime}$. These variables are not independent, because of the relations $\dot{\Phi}=a_{\mu} \dot{x}^{\mu}$ and $\Phi^{\prime}=a_{\mu} x^{\mu \prime}$, which are equivalent to the following connection between the currents $i_{ \pm}^{\Phi}=2 a^{\mu} J_{ \pm \mu}$. The last one can be confirmed directly from (2.15). 
After long but straightforward calculation, the canonical Hamiltonian density obtains the form

$$
\mathcal{H}_{c}=h^{-} T_{-}+h^{+} T_{+}+q^{\prime},
$$

where the expressions for the energy momentum tensor components

$$
T_{ \pm}=\mp \frac{1}{4 \kappa}\left(G^{\mu \nu} J_{ \pm \mu} J_{ \pm \nu}+i_{ \pm}^{F} i_{ \pm}^{\Phi}\right)+\frac{1}{2} i_{ \pm}^{\Phi \prime}=\mp \frac{1}{4 \kappa}\left(G^{\mu \nu} j_{ \pm \mu} j_{ \pm \nu}-\frac{j^{2}}{a^{2}}\right)+i_{ \pm}
$$

contains the useful combination of the currents

$$
i_{ \pm}=\frac{1}{2}\left(i_{ \pm}^{\Phi^{\prime}}-F^{\prime} i_{ \pm}^{\Phi}\right)
$$

In the expression for the canonical Hamiltonian $H_{c}=\int d \sigma \mathcal{H}_{c}$ the term

$$
q=-\frac{1}{2}\left(h^{-} i_{-}^{\Phi}+h^{+} i_{+}^{\Phi}\right)
$$

appears only on the world-sheet boundary $\partial \Sigma$ and does not contribute to the equations of motion.

\section{Virasoro algebra in the curved space-time}

We shall start with basic Poisson brackets (PB) algebra

$$
\left\{x^{\mu}, \pi_{\nu}\right\}=\delta_{\nu}^{\mu} \delta
$$

Through the paper, we will understand that the first variable in PB depends on $\sigma$ and the second one on $\bar{\sigma}$. We will also use the conventions $\delta \equiv \delta(\sigma-\bar{\sigma}), \delta^{\prime} \equiv \partial_{\sigma} \delta(\sigma-\bar{\sigma})$ and $\delta^{\prime \prime} \equiv \partial_{\sigma}^{2} \delta(\sigma-\bar{\sigma})$. On the right hand side, all $\bar{\sigma}$ dependence we will write explicitly and $\sigma$ dependence we will omit.

Let us first calculate PB between the currents $j_{ \pm \mu}, i_{ \pm}^{\Phi}, j$ and $i_{ \pm}$

$$
\begin{gathered}
\left\{j_{ \pm \mu}, j_{ \pm \nu}\right\}= \pm 2 \kappa\left(G_{\mu \nu} \delta^{\prime}+\Gamma_{\mp \mu, \nu \rho} x^{\rho \prime} \delta\right), \quad\left\{j_{ \pm \mu}, j_{\mp \nu}\right\}= \pm 2 \kappa \Gamma_{\mp \rho, \mu \nu} x^{\prime \rho} \delta \\
\left\{j_{ \pm \mu}, i_{ \pm}^{\Phi}\right\}= \pm 2 \kappa a_{\mu} \delta^{\prime}, \quad\left\{j_{ \pm \mu}, i_{\mp}^{\Phi}\right\}=\mp 2 \kappa a_{\mu} \delta^{\prime} \\
\left\{i_{ \pm}^{\Phi}, i_{ \pm}^{\Phi}\right\}=0, \quad\left\{i_{ \pm}^{\Phi}, i_{\mp}^{\Phi}\right\}=0 \\
\{j, j\}=0, \quad\left\{j, i_{ \pm}^{\Phi}\right\}= \pm 2 \kappa a^{2} \delta^{\prime}, \quad\left\{j, i_{ \pm}\right\}=\mp \kappa a^{2}\left[\delta^{\prime \prime}+F^{\prime}(\bar{\sigma}) \delta^{\prime}\right]+\frac{1}{4} i_{ \pm}^{\Phi}(\bar{\sigma}) \delta^{\prime}, \\
\left\{i_{ \pm}, i_{ \pm}\right\}=-\frac{1}{2}\left[i_{ \pm}(\sigma)+i_{ \pm}(\bar{\sigma})\right] \delta^{\prime}, \quad\left\{i_{ \pm}, i_{\mp}\right\}=-\frac{1}{2}\left[i_{\mp}(\sigma)+i_{\mp}(\bar{\sigma})\right] \delta^{\prime} \pm \kappa \Phi^{\prime}\left(\delta^{\prime \prime}+F^{\prime} \delta^{\prime}+F^{\prime \prime} \delta\right) \\
\left\{i_{ \pm}, i_{ \pm}^{\Phi}\right\}=-\frac{1}{2} i_{ \pm}^{\Phi} \delta^{\prime}, \quad\left\{i_{ \pm}, i_{\mp}^{\Phi}\right\}=-\frac{1}{2} i_{ \pm}^{\Phi} \delta^{\prime}
\end{gathered}
$$


The expressions $\Gamma_{ \pm \rho, \mu \nu}$ are defined as

$$
\Gamma_{ \pm \nu \mu}^{\rho}=\Gamma_{\nu \mu}^{\rho} \pm B_{\nu \mu}^{\rho}
$$

where the first term is Christoffel connection

$$
\Gamma_{\nu \mu}^{\rho}=\frac{1}{2} G^{\rho \sigma}\left(\partial_{\nu} G_{\mu \sigma}+\partial_{\mu} G_{\nu \sigma}-\partial_{\sigma} G_{\nu \mu}\right)
$$

and the second one is the antisymmetric tensor field strength

$$
B_{\mu \nu \rho}=\partial_{\mu} B_{\nu \rho}+\partial_{\nu} B_{\rho \mu}+\partial_{\rho} B_{\mu \nu}=D_{\mu} B_{\nu \rho}+D_{\nu} B_{\rho \mu}+D_{\rho} B_{\mu \nu}
$$

The "generalized connection" is expressed in terms of the variables $G_{ \pm \mu \nu}= \pm 2 \Pi_{ \pm \mu \nu}=$ $G_{\mu \nu} \pm 2 B_{\mu \nu}$ in the similar way as Christoffel connection is expressed in terms of $G_{\mu \nu}$

$$
\Gamma_{ \pm \rho, \nu \mu}=\frac{1}{2}\left(\partial_{\nu} G_{ \pm \mu \rho}+\partial_{\mu} G_{ \pm \rho \nu}-\partial_{\rho} G_{ \pm \mu \nu}\right) .
$$

For any momenta independent variables, $X$, we have

$$
\left\{X, T_{ \pm}\right\}=\mp \frac{1}{2 \kappa} J_{ \pm}^{\mu} \partial_{\mu} X \delta .
$$

Let us write the expression for the energy-momentum tensor components in the form $T_{ \pm}=t_{ \pm} \pm t$, where

$$
t_{ \pm}=\mp \frac{1}{4 \kappa} G^{\mu \nu} j_{ \pm \mu} j_{ \pm \nu}+i_{ \pm}, \quad t=\frac{1}{4 \kappa} \frac{j^{2}}{a^{2}}
$$

and let us introduce the combination

$$
\tau=\frac{1}{2}\left(j^{\prime}-F^{\prime} j\right)
$$

Then we obtain the following algebra

$$
\begin{gathered}
\left\{t_{ \pm}, t_{ \pm}\right\}=-\left[t_{ \pm}(\sigma)+t_{ \pm}(\bar{\sigma})\right] \delta^{\prime}-[\tau(\sigma)+\tau(\bar{\sigma})] \delta^{\prime} \\
\left\{t_{ \pm}, t\right\}+\left\{t, t_{ \pm}\right\}=-[t(\sigma)+t(\bar{\sigma})] \delta^{\prime} \pm[\tau(\sigma)+\tau(\bar{\sigma})] \delta^{\prime} \\
\{t, t\}=0
\end{gathered}
$$

which produce the Virasoro algebra for the same chirality energy-momentum tensor components

$$
\left\{T_{ \pm}, T_{ \pm}\right\}=-\left[T_{ \pm}(\sigma)+T_{ \pm}(\bar{\sigma})\right] \delta^{\prime} .
$$

Similarly, for the opposite chiralities we have

$$
\left\{t_{ \pm}, t_{\mp}\right\}=[\tau(\sigma)+\tau(\bar{\sigma})] \delta^{\prime}
$$




$$
\left\{t_{ \pm}, t\right\}-\left\{t, t_{ \pm}\right\}= \pm[\tau(\sigma)+\tau(\bar{\sigma})] \delta^{\prime}
$$

which contribute to the fact that the opposite chirality energy-momentum tensor components commute

$$
\left\{T_{ \pm}, T_{\mp}\right\}=0 .
$$

Consequently, the expression (2.21) presents new, non-linear realization of the Virasoro algebras. The dilaton field $\Phi$ is the origin of the non-linearity. We want to stress, that the opposite chirality currents generally do not commute in the curved space time, see (3.2)-(3.7), but the opposite chirality energy-momentum tensors components commute.

The momenta $\pi_{ \pm}$, defined in (2.6), are primary constraints. The total Hamiltonian takes the form $H_{T}=\int d \sigma\left(\mathcal{H}_{c}+\lambda^{-} \pi_{-}+\lambda^{+} \pi_{+}\right)$. The consistency conditions $\dot{\pi}_{ \pm}=$ $\left\{\pi_{ \pm}, H_{T}\right\}=-T_{ \pm}$produce the secondary constraints $T_{ \pm}$. As a consequence of the Virasoro algebra (3.18) and (3.21), the consistency conditions for the secondary constraints $T_{ \pm}$are satisfied and there are no more constraints. All constraints are first class and they are generators of two dimensional diffeomorphisms [5]. The canonical Hamiltonian is weakly equal to zero.

\section{Equations of motion}

Using the expressions for the energy momentum tensor and for the currents obtained in the previous subsections, we are going to find equations of motion for the action (2.1). First we obtain Hamiltonian equations of motion and then the corresponding Lagrangian ones. It is useful to define the expression $V_{ \pm}=V^{\mu}(x) J_{ \pm \mu}$, where $V^{\mu}(x)$ is an arbitrary vector function of the coordinates $x^{\mu}$. Then with the help of (2.20) and (2.21) we have

$$
\dot{V}_{ \pm}=\left\{V_{ \pm}, H_{c}\right\}=-\left(h^{ \pm} V_{ \pm}\right)^{\prime}+\frac{h^{-}-h^{+}}{2 \kappa}\left({ }^{\star} D_{\mp \mu} V_{\nu}\right) J_{ \pm}^{\nu} J_{\mp}^{\mu},
$$

where we introduce notation

$$
\begin{gathered}
{ }^{\star} D_{ \pm \mu} V_{\nu}=\partial_{\mu} V_{\nu}-{ }^{\star} \Gamma_{ \pm \nu \mu}^{\rho} V_{\rho}=D_{ \pm \mu} V_{\nu}-\frac{a^{\rho} V_{\rho}}{a^{2}} D_{ \pm \mu} a_{\nu} \\
{ }^{\star} \Gamma_{ \pm \nu \mu}^{\rho}=\quad \Gamma_{ \pm \nu \mu}^{\rho}+\frac{a^{\rho}}{a^{2}} D_{ \pm \mu} a_{\nu}=P^{T \rho}{ }_{\sigma} \Gamma_{ \pm \nu \mu}^{\sigma}+\frac{a^{\rho}}{a^{2}} \partial_{\mu} a_{\nu}=\Gamma_{\nu \mu}^{\rho} \pm P^{T \rho}{ }_{\sigma} B_{\nu \mu}^{\sigma}+\frac{a^{\rho}}{a^{2}} D_{\mu} a_{\nu}
\end{gathered}
$$

Under space-time general coordinate transformations the expression ${ }^{\star} \Gamma_{ \pm \nu \mu}^{\rho}$ transforms as a connection. It contains the Christoffel connection (3.9), itself. If we consider ${ }^{\star} \Gamma_{ \pm \nu \mu}^{\rho}$ as a space-time connection, then ${ }^{\star} D_{ \pm \mu}$ is corresponding covariant derivative. The consequences of such approach we will explain in detail in the next paper [7].

We can rewrite (4.1) in the world-sheet covariant way

$$
\hat{\nabla}_{\mp} V_{ \pm}=\frac{1}{\sqrt{2} \kappa}\left({ }^{\star} D_{\mp \mu} V_{\nu}\right) J_{ \pm}^{\nu} J_{\mp}^{\mu} .
$$


For $V_{\mu}=$ const, the last equation produce the equation of motion for the current $J_{ \pm}^{\mu}$

$$
\hat{\nabla}_{\mp} J_{ \pm}^{\mu}+\frac{1}{\sqrt{2} \kappa}{ }^{\star} \Gamma_{\mp \rho \sigma}^{\mu} J_{ \pm}^{\rho} J_{\mp}^{\sigma}=0
$$

and for $V_{\mu}=a_{\mu}$, the equation of motion for the current $i_{ \pm}^{\Phi}$

$$
\hat{\nabla}_{\mp} i_{ \pm}^{\Phi}=0
$$

From (4.3) and (4.4) we also obtain

$$
\hat{\partial}_{\mp} V_{\mu}=\frac{1}{\sqrt{2} \kappa} \partial_{\nu} V_{\mu} J_{\mp}^{\nu} .
$$

Similarly, the equation of motion for the current $i_{ \pm}^{F}$ has the form

$$
\hat{\nabla}_{\mp} i_{ \pm}^{F} \pm \frac{2 \sqrt{2} \kappa}{h^{-}-h^{+}} h^{ \pm \prime \prime}=\frac{1}{\sqrt{2} \kappa a^{2}}\left(D_{\mp \mu} a_{\nu}\right) J_{ \pm}^{\nu} J_{\mp}^{\mu}
$$

So, (4.4), (4.5) and (4.7) are Hamiltonian equations of motion. The equations for the momenta $\pi_{ \pm}$produce the vanishing of the energy momentum tensor components $T_{ \pm}=0$.

To obtain corresponding Lagrangian equations, it is possible to substitute the expressions for the momenta into the Hamiltonian equations, but there is equivalent and simpler approach. First we obtain the Lagrangian expressions for the currents

$$
J_{ \pm \mu}=\sqrt{2} \kappa G_{\mu \nu} \hat{\partial}_{ \pm} x^{\nu}, \quad i_{ \pm}^{\Phi}=2 \sqrt{2} \kappa \hat{\partial}_{ \pm} \Phi, \quad i_{ \pm}^{F}=\sqrt{2} \kappa\left(2 \hat{\partial}_{ \pm} F \mp \hat{\omega}_{ \pm} \pm \hat{\omega}_{\mp}\right),
$$

solving linear system of the equations, (2.14), (2.8), (2.11) and (2.19) and eliminating $\tau$ and $\sigma$ derivatives of the corresponding variables. Then, we substitute them into Hamiltonian equations and find

$$
\begin{gathered}
\hat{\Delta} x^{\mu}-2^{\star} \Gamma_{\mp \rho \sigma}^{\mu} \hat{\partial}_{ \pm} x^{\rho} \hat{\partial}_{\mp} x^{\sigma}=0, \\
\hat{\Delta} \Phi=0 \\
\hat{\Delta} F+\frac{1}{2} \hat{R}^{(2)}+\frac{1}{a^{2}}\left(D_{\mp \mu} a_{\nu}\right) \hat{\partial}_{ \pm} x^{\nu} \hat{\partial}_{\mp} x^{\mu}=0 \\
T_{ \pm}=\mp \frac{\kappa}{2}\left(G_{\mu \nu} \hat{\partial}_{ \pm} x^{\mu} \hat{\partial}_{ \pm} x^{\nu}-2 \hat{\nabla}_{ \pm} \hat{\partial}_{ \pm} \Phi+4 \hat{\partial}_{ \pm} F \hat{\partial}_{ \pm} \Phi-\hat{\Delta} \Phi\right)=0 .
\end{gathered}
$$

Note that equations (4.9) (with + and - indices) are equivalent, because of the property ${ }^{\star} \Gamma_{ \pm \rho \sigma}^{\mu}={ }^{\star} \Gamma_{\mp \sigma \rho}^{\mu}$, as well as equations (4.11) are equivalent, because of the property $D_{ \pm \mu} a_{\nu}=D_{\mp \nu} a_{\mu}$.

The Lagrangian expression for the equation (4.6) produce just the chain rule

$$
\hat{\partial}_{ \pm} V_{\mu}=\hat{\partial}_{ \pm} x^{\nu} \partial_{\nu} V_{\mu}
$$


As a consequence of the relation $i_{ \pm}^{\Phi}=2 a^{\mu} J_{ \pm \mu}$, the equation (4.10) follows from (4.9) and can be obtained just multiplying the last one by $a_{\mu}$.

Finally, we can go from the hat variables to the original ones (see App. A) and obtain the final form of the Lagrangian field equations

$$
\begin{gathered}
\nabla_{\mp} \partial_{ \pm} x^{\mu}+{ }^{\star} \Gamma_{\mp \rho \sigma}^{\mu} \partial_{ \pm} x^{\rho} \partial_{\mp} x^{\sigma}=0, \\
G_{\mu \nu} \partial_{ \pm} x^{\mu} \partial_{ \pm} x^{\nu}-2 \nabla_{ \pm} \partial_{ \pm} \Phi=0 \\
R^{(2)}+\frac{2}{a^{2}}\left(D_{\mp \mu} a_{\nu}\right) \partial_{ \pm} x^{\nu} \partial_{\mp} x^{\mu}=0 .
\end{gathered}
$$

\section{Conclusions and discussion}

In this paper, we investigated bosonic string theory in presence of background fields. We used the Hamiltonian method and treated the space-time coordinates $x^{\mu}$ and the world-sheet metric $g_{\alpha \beta}$ as variables in the theory. We did the analysis for the arbitrary background fields as functions of the string coordinates.

We obtained a new, nonlinear realization of the energy-momentum tensor components (2.21). They satisfy two independent Virasoro algebras, which is expected because the theory is invariant under world-sheet reparametrization. These components are bilinear in the currents or linear in the $\sigma$ derivative of the currents. All currents are linear in the momenta and in the $\sigma$ derivative of the fields. Let us stress that the opposite chirality components of the energy-momentum tensor commute, (3.21), in spite of the fact that the corresponding currents do not commute. Using appropriate commutation relations we obtain the Hamiltonian equations of motion which easily produce the Lagrangian ones.

The equations of motion (4.14)-(4.16) offer the possibility to investigate the dynamics of the string, propagating in the curved target space. Starting with the given stringy connection (4.2), let us here shortly discuss the main features of the target space geometry. The space-time observed by the string moving in the background $G_{\mu \nu}, B_{\mu \nu}$ and $\Phi$, we will call stringy space-time.

The antisymmetric part of the stringy connection is the stringy torsion

$$
{ }^{\star} T_{ \pm} \stackrel{\rho}{\rho} \equiv{ }^{\star} \Gamma_{ \pm} \stackrel{\rho}{\mu \nu}-{ }^{\star} \Gamma_{ \pm}{ }_{\nu \mu}^{\rho}= \pm 2 P^{T \rho}{ }_{\sigma} B_{\mu \nu}^{\sigma},
$$

which is the transverse part of the field strength of the antisymmetric tensor field $B_{\mu \nu}$.

We define the stringy parallel transport as usual

$$
{ }^{\star} \delta_{ \pm} V^{\mu}=-{ }^{\star} \Gamma_{ \pm \rho \sigma}^{\mu} V^{\rho} d x^{\sigma} .
$$

The metric postulate is defined by the demand, that after parallel transport the metric is equal to the local one. The most interesting feature of the stringy geometry is the breaking 
of the space-time metric postulate. The stringy nonmetricity

$$
{ }^{\star} Q_{ \pm \mu \rho \sigma} \equiv-{ }^{\star} D_{ \pm \mu} G_{\rho \sigma}=\frac{1}{a^{2}} D_{ \pm \mu}\left(a_{\rho} a_{\sigma}\right)
$$

is different from zero, which means that metric $G_{\mu \nu}$ is not compatible with stringy connection ${ }^{\star} \Gamma_{ \pm \nu \rho}^{\mu}$. Consequently, during parallel transport the length and angle deformations depend on the vector field $a_{\mu}$.

So, with the help of the equations of motion, we recognize the expressions ${ }^{\star} \Gamma_{ \pm \mu \nu}^{\rho}$ as stringy connections. It helps us to conclude that the string can feel the space-time torsion and nonmetricity. The more detailed investigation of the stringy geometry and geometrical interpretation of the equations of motion will be presented in [7].

\section{A World-sheet geometry in the light-cone basis}

In this appendix we present our notations and conventions concerning intrinsic world sheet geometry in the light-cone basis.

We parameterize the world-sheet metric $g_{\alpha \beta}$ with light-cone variables $\left(h^{+}, h^{-}, F\right)$ [5, 6]

$$
g_{\alpha \beta}=e^{2 F} \hat{g}_{\alpha \beta}=\frac{1}{2} e^{2 F}\left(\begin{array}{cc}
-2 h^{-} h^{+} & h^{-}+h^{+} \\
h^{-}+h^{+} & -2
\end{array}\right) .
$$

The interval of the world-sheet $\Sigma$

$$
d s^{2}=g_{\alpha \beta} d \xi^{\alpha} d \xi^{\beta}=2 d \xi^{+} d \xi^{-},
$$

can be expressed in terms of the light-cone coordinates

$$
d \xi^{ \pm}=\frac{ \pm 1}{\sqrt{2}} e^{F}\left(d \xi^{1}-h^{ \pm} d \xi^{0}\right)=e^{F} d \hat{\xi}^{ \pm}=e^{ \pm}{ }_{\alpha} d \xi^{\alpha} .
$$

The quantities $e^{ \pm}{ }_{\alpha}$ define the light-cone one form basis $\theta^{ \pm}=e^{ \pm}{ }_{\alpha} d \xi^{\alpha}$, and its inverse define the tangent vector basis $e_{ \pm}=e_{ \pm}{ }^{\alpha} \partial_{\alpha}=\partial_{ \pm}$. In this basis, components of the arbitrary vector $V_{\alpha}$ are

$$
V_{ \pm}=e^{-F} \hat{V}_{ \pm}=e_{ \pm}^{\alpha} V_{\alpha}=\frac{\sqrt{2} e^{-F}}{h^{-}-h^{+}}\left(V_{0}+h^{\mp} V_{1}\right)
$$

The world-sheet covariant derivatives on tensor $X_{n}$ are

$$
\nabla_{ \pm} X_{n}=\left(\partial_{ \pm}+n \omega_{ \pm}\right) X_{n}
$$

where the number $n$ is sum of the indices, counting index + with 1 and index - with -1 , and

$$
\omega_{ \pm}=e^{-F}\left(\hat{\omega}_{ \pm} \mp \hat{\partial}_{ \pm} F\right), \quad \hat{\omega}_{ \pm}=\mp \frac{\sqrt{2}}{h^{-}-h^{+}} h^{\mp \prime}
$$


are two dimensional Riemannian connections. In this notations the world-sheet scalar curvature has the form

$$
R^{(2)}=2 \nabla_{-} \omega_{+}-2 \nabla_{+} \omega_{-} .
$$

We also use the relation

$$
\sqrt{-g} R^{(2)}=\sqrt{-\hat{g}}\left(\hat{R}^{(2)}+2 \hat{\Delta} F\right)
$$

where

$$
\hat{\Delta}=-2 \hat{\nabla}_{ \pm} \hat{\partial}_{\mp}
$$

is the Laplace operator.

\section{References}

[1] M. B. Green, J. H. Schwarz and E. Witten, Superstring Theory, Cambridge University Press, 1987.

[2] E. S. Fradkin and A. A. Tseytlin, Phys. Lett. B 158 (1985) 316; Nucl. Phys. B 261 (1985) 1.

[3] C. G. Callan, D. Friedan, E. J. Martinec and M. J. Perry, Nucl. Phys. B 262 (1985) 593.

[4] T. Banks, D. Nemeschansky and A. Sen, Nucl. Phys. B 277 (1986) 67.

[5] M. Blagojević, D. S. Popović and B. Sazdović, Mod. Phys. Lett. A 13 (1998) 911; Phys. Rev. D 59 (1999) 044021; D. S. Popović and B. Sazdović, Mod. Phys. Lett. A 16 (2001) 589.

[6] B. Sazdović, Phys. Rev. D 59 (1999) 084008; O. Mišković and B. Sazdović, Mod. Phys. Lett. A 17 (2002) 1923.

[7] B. Sazdović, Torsion and nonmetricity in the stringy geometry, hep-th/0304086 